\title{
Vertical and Adiabatic Ionization Potentials of Fluorinated, Chlorinated and Chlorofluorinated Ethylenes using G2 and G3 Theories
}

\author{
Noriberto A. Pradie, Harrald V. Linnert \\ Instituto de Química, Universidade de São Paulo, Caixa Postal 26077, CEP 05513-970 São Paulo, SP, \\ Brazil; hvlinner@iq.usp.br, noriberto@yahoo.com
}

On the following pages are included:

a) the calculated energies obtained at G2 and G3 theories (Table S1), ZPE (Table S2) and Hartree Fock energies (TableS3) of all neutral and charged subtances, and the experimental ionization potentials (Table S4).

b) results obtained with $\mathrm{G} 2$ theory to $-\varepsilon, \mathrm{Er}_{\mathrm{n}-1}^{\mathrm{CN}}, \Delta \mathrm{E}(\text { corr })_{\mathrm{n}, \mathrm{n}-1}^{\mathrm{CN}}, \Delta \mathrm{ZPE}_{\mathrm{n}, \mathrm{n}-1}^{\mathrm{CN}}, \operatorname{VIP}(\mathrm{GX}), \mathrm{E}_{\mathrm{r}}(\mathrm{nucl})_{\mathrm{n}-1}$, $\Delta \mathrm{E}(\operatorname{corr})_{\mathrm{n}-1, \mathrm{n}-1}^{\mathrm{RN}}, \Delta \mathrm{ZPE} \mathrm{E}_{\mathrm{n}-1, \mathrm{n}-1}^{\mathrm{RN}}$ and $\mathrm{AIP}(\mathrm{G} 2)$ as a function of number of halogens (Figures $\mathrm{S} 1$ to $\mathrm{S} 3, \mathrm{~S} 5$ to $\mathrm{S} 7, \mathrm{~S} 9, \mathrm{~S} 10, \mathrm{~S} 12$ and S13), the relationship between VIP(G2) and $-\varepsilon$ (Figure S8), $\mathrm{Er}_{\mathrm{n}-1}^{\mathrm{CN}}+\Delta \mathrm{E}(\mathrm{corr})_{\mathrm{n}, \mathrm{n}-1}^{\mathrm{CN}}$ and $\mathrm{E}_{\mathrm{r}}(\mathrm{nucl})_{\mathrm{n}-1}+\Delta \mathrm{E}(\mathrm{corr})_{\mathrm{n}-1, \mathrm{n}-1}^{\mathrm{RN}}$ as a function of the number of halogens (Figure S4 and S11 respectively), and

c) the complete citation for reference 13 and 14 : 
Table S1. G2(0K) and G3(0K) energies obtained by G2 and G3 theories

\begin{tabular}{|c|c|c|c|c|c|c|}
\hline \multirow[b]{2}{*}{ substance } & \multicolumn{3}{|c|}{$\mathrm{E}(\mathrm{G} 2) /$ Hartree } & \multicolumn{3}{|c|}{$\mathrm{E}(\mathrm{G} 3) /$ Hartree } \\
\hline & neutral & $\begin{array}{c}\text { positive } \\
\text { relaxed nuclei }\end{array}$ & $\begin{array}{c}\text { positive } \\
\text { coupled nuclei }\end{array}$ & neutral & $\begin{array}{c}\text { positive } \\
\text { relaxed nuclei }\end{array}$ & $\begin{array}{c}\text { positive } \\
\text { coupled nuclei }\end{array}$ \\
\hline $\mathrm{CH} 2=\mathrm{CH} 2$ & $-78,415927$ & $-78,028137$ & $-78,020829$ & $-78,507417$ & $-78,120294$ & $-78,113473$ \\
\hline $\mathrm{CH} 2=\mathrm{CHCl}$ & -537.568284 & -537.201103 & -537.194132 & -537.972234 & -537.60529 & -537.598217 \\
\hline $\mathrm{E}-\mathrm{CHCl}=\mathrm{CHCl}$ & -996.717299 & -996.364558 & -996.356249 & -997.433641 & -997.08107 & -997.072476 \\
\hline $\mathrm{Z}-\mathrm{CHCl}=\mathrm{CHCl}$ & -996.717906 & -996.363978 & -996.356091 & -997.434343 & -997.08071 & -997.072559 \\
\hline $\mathrm{E}-\mathrm{CHCl}=\mathrm{CFCl}$ & -1095.868594 & -1095.515851 & -1095.505156 & -1096.633815 & -1096.2813 & -1096.27035 \\
\hline $\mathrm{Z}-\mathrm{CHCl}=\mathrm{CFCl}$ & -1095.868224 & -1095.515265 & -1095.504285 & -1096.633591 & -1096.2809 & -1096.2696 \\
\hline $\mathrm{CH} 2=\mathrm{CHF}$ & -177.572130 & -177.190903 & -177.178961 & -177.712566 & -177.33153 & -177.319861 \\
\hline $\mathrm{Z}-\mathrm{CHF}=\mathrm{CHF}$ & -276.718160 & -276.342780 & -276.326544 & -276.907308 & -276.53192 & -276.515867 \\
\hline $\mathrm{E}-\mathrm{CHF}=\mathrm{CHF}$ & -276.716996 & -276.342917 & -276.326093 & -276.906311 & -276.53206 & -276.515376 \\
\hline $\mathrm{E}-\mathrm{CFCl}=\mathrm{CHF}$ & -735.866441 & -735.504141 & -735.489360 & -736.368061 & -736.00582 & -735.990905 \\
\hline $\mathrm{Z}-\mathrm{CFCl}=\mathrm{CHF}$ & -735.866470 & -735.504598 & -735.489818 & -736.368221 & -736.00643 & -735.991561 \\
\hline $\mathrm{E}-\mathrm{CFCl}=\mathrm{CFCl}$ & -1195.014373 & -1194.662574 & -1194.649331 & -1195.828493 & -1195.477 & -1195.46348 \\
\hline $\mathrm{Z}-\mathrm{CFCl}=\mathrm{CFCl}$ & -1195.013550 & -1194.661122 & -1194.647699 & -1195.827697 & -1195.4756 & -1195.46182 \\
\hline $\mathrm{CF} 2=\mathrm{CFCl}$ & -835.018613 & -834.658024 & -834.640883 & -835.569264 & -835.2088 & -835.191466 \\
\hline $\mathrm{CF} 2=\mathrm{CF} 2$ & -475.022042 & -474.650838 & -474.627773 & -475.309177 & -474.93781 & -474.91475 \\
\hline $\mathrm{CCl} 2=\mathrm{CCl} 2$ & -1915.010878 & -1914.669215 & -1914.660709 & -1916.353152 & -1916.0118 & -1916.00279 \\
\hline $\mathrm{CCl} 2=\mathrm{CF} 2$ & -1195.019179 & -1194.665862 & -1194.652869 & -1195.833705 & -1195.4805 & -1195.46721 \\
\hline $\mathrm{CCl} 2=\mathrm{CHCl}$ & -1455.865264 & -1455.512692 & -1455.509555 & -1456.894473 & -1456.5476 & -1456.53862 \\
\hline $\mathrm{CF} 2=\mathrm{CHF}$ & -375.872587 & -375.500313 & -375.480042 & -376.110799 & -375.73838 & -375.718186 \\
\hline $\mathrm{CCl} 2=\mathrm{CFCl}$ & -1555.013271 & -1554.66076 & -1554.656472 & -1556.091401 & -1555.7454 & -1555.7345 \\
\hline $\mathrm{CCl} 2=\mathrm{CH} 2$ & -996.717245 & -996.357138 & -996.348598 & -997.433978 & -997.07397 & -997.065343 \\
\hline $\mathrm{CF} 2=\mathrm{CH} 2$ & -276.733531 & -276.355064 & -276.338774 & -276.922991 & -276.54459 & -276.528509 \\
\hline $\mathrm{CHCl}=\mathrm{CF} 2$ & -735.875834 & -735.515188 & -735.501430 & -736.377694 & -736.01716 & -736.003209 \\
\hline $\mathrm{CCl} 2=\mathrm{CHF}$ & -1095.864827 & -1095.510119 & -1095.499038 & -1096.630374 & -1096.2757 & -1096.26442 \\
\hline $\mathrm{CFCl}=\mathrm{CH} 2$ & -636.723225 & -636.355293 & -636.343889 & -637.176129 & -636.8084 & -636.796956 \\
\hline $\mathrm{Z}-\mathrm{CHCl}=\mathrm{CHF}$ & -636.718959 & -636.356108 & -636.345624 & -637.171772 & -636.80907 & -636.798502 \\
\hline $\mathrm{E}-\mathrm{CHCl}=\mathrm{CHF}$ & -636.717974 & -636.35605 & -636.344797 & -637.170834 & -636.80891 & -636.797522 \\
\hline
\end{tabular}


Table S2. calculated ZPE (Hartree Fock value corrected by 0.8929 scale factor) obtained by G2 and G3 theories

\begin{tabular}{|c|c|c|c|c|c|c|c|}
\hline \multirow{2}{*}{ substance } & \multirow{2}{*}{ symmetry } & \multicolumn{3}{|c|}{ ZPE(G2)/ Hartree } & \multicolumn{3}{|c|}{ ZPE(G3)/ Hartree } \\
\hline & & neutral & $\begin{array}{c}\text { positive } \\
\text { coupled nucle }\end{array}$ & $\begin{array}{c}\text { positive } \\
\text { relaxed nuclei }\end{array}$ & neutral & $\begin{array}{c}\text { positive } \\
\text { coupled nucl }\end{array}$ & $\begin{array}{c}\text { positive } \\
\text { elaxed nuclei }\end{array}$ \\
\hline $\mathrm{CH} 2=\mathrm{CH} 2$ & $\mathrm{D}_{2 \mathrm{H}} *$ & 0,048909 & 0,047538 & 0.046827 & 0,048903 & 0,047539 & 0.046827 \\
\hline $\mathrm{CH} 2=\mathrm{CHCl}$ & $\mathrm{C}_{\mathrm{S}}$ & 0.041204 & 0.040131 & 0.040282 & 0.041204 & 0.040131 & 0.040282 \\
\hline $\mathrm{E}-\mathrm{CHCl}=\mathrm{CHCl}$ & $\mathrm{C}_{2 \mathrm{H}}$ & 0.032956 & 0.032289 & 0.032540 & 0.032956 & 0.032289 & 0.032540 \\
\hline $\mathrm{Z}-\mathrm{CHCl}=\mathrm{CHCl}$ & $\mathrm{C}_{2 \mathrm{~V}}$ & 0.033274 & 0.03221 & 0.032754 & 0.033274 & 0.03221 & 0.032754 \\
\hline $\mathrm{E}-\mathrm{CHCl}=\mathrm{CFCl}$ & $\mathrm{C}_{\mathrm{S}}$ & 0.025865 & 0.024947 & 0.025577 & 0.025865 & 0.024947 & 0.025577 \\
\hline $\mathrm{Z}-\mathrm{CHCl}=\mathrm{CFCl}$ & $\mathrm{C}_{\mathrm{S}}$ & 0.025786 & 0.024833 & 0.025480 & 0.025786 & 0.024833 & 0.025480 \\
\hline $\mathrm{CH} 2=\mathrm{CHF}$ & $\mathrm{C}_{\mathrm{S}}$ & 0.042549 & 0.041319 & 0.041748 & 0.042549 & 0.041319 & 0.041748 \\
\hline $\mathrm{Z}-\mathrm{CHF}=\mathrm{CHF}$ & $\mathrm{C}_{2 \mathrm{~V}}$ & 0.036107 & 0.034773 & 0.035911 & 0.036107 & 0.034773 & 0.035911 \\
\hline $\mathrm{E}-\mathrm{CHF}=\mathrm{CHF}$ & $\mathrm{C}_{2 \mathrm{H}}$ & 0.035753 & 0.034762 & 0.035561 & 0.035753 & 0.034762 & 0.035561 \\
\hline $\mathrm{E}-\mathrm{CFCl}=\mathrm{CHF}$ & $\mathrm{C}_{\mathrm{S}}$ & 0.027291 & 0.026270 & 0.027135 & 0.027291 & 0.026271 & 0.027135 \\
\hline $\mathrm{Z}-\mathrm{CFCl}=\mathrm{CHF}$ & $\mathrm{C}_{\mathrm{S}}$ & 0.02726 & 0.026167 & 0.027063 & 0.02726 & 0.026167 & 0.027063 \\
\hline $\mathrm{E}-\mathrm{CFCl}=\mathrm{CFCl}$ & $\mathrm{C}_{2 \mathrm{H}}$ & 0.018362 & 0.017566 & 0.018477 & 0.018362 & 0.017566 & 0.018477 \\
\hline $\mathrm{Z}-\mathrm{CFCl}=\mathrm{CFCl}$ & $\mathrm{C}_{2 \mathrm{~V}}$ & 0.018313 & 0.017547 & 0.018464 & 0.018313 & 0.017549 & 0.018464 \\
\hline $\mathrm{CF} 2=\mathrm{CFCl}$ & $\mathrm{C}_{\mathrm{S}}$ & 0.019936 & 0.019018 & 0.020238 & 0.019934 & 0.019018 & 0.020238 \\
\hline $\mathrm{CF} 2=\mathrm{CF} 2$ & $\mathrm{D}_{2 \mathrm{H}}$ & 0.021408 & 0.020379 & 0.021939 & 0.021408 & 0.020379 & 0.021941 \\
\hline $\mathrm{CCl} 2=\mathrm{CCl} 2$ & $\mathrm{D}_{2 \mathrm{H}}$ & 0.015362 & 0.014916 & 0.015208 & 0.015361 & 0.014915 & 0.015208 \\
\hline $\mathrm{CCl} 2=\mathrm{CF} 2$ & $\mathrm{C}_{2 \mathrm{~V}}$ & 0.018628 & 0.017855 & 0.018724 & 0.018628 & 0.017855 & 0.018724 \\
\hline $\mathrm{CCl} 2=\mathrm{CHCl}$ & $\mathrm{C}_{\mathrm{S}}$ & 0.024391 & 0.023643 & 0.023980 & 0.024391 & 0.023643 & 0.023980 \\
\hline $\mathrm{CF} 2=\mathrm{CHF}$ & $\mathrm{C}_{\mathrm{S}}$ & 0.028782 & 0.027544 & 0.028772 & 0.028783 & 0.027544 & 0.028772 \\
\hline $\mathrm{CCl} 2=\mathrm{CFCl}$ & $\mathrm{C}_{\mathrm{S}}$ & 0.016909 & 0.016295 & 0.016878 & 0.016913 & 0.016295 & 0.016878 \\
\hline $\mathrm{CCl} 2=\mathrm{CH} 2$ & $\mathrm{C}_{2 \mathrm{~V}}$ & 0.032731 & 0.031555 & 0.031686 & 0.032731 & 0.031555 & 0.031685 \\
\hline $\mathrm{CF} 2=\mathrm{CH} 2$ & $\mathrm{C}_{2 \mathrm{~V}}$ & 0.035620 & 0.034049 & 0.034861 & 0.035620 & 0.034049 & 0.034861 \\
\hline $\mathrm{CHCl}=\mathrm{CF} 2$ & $\mathrm{C}_{\mathrm{S}}$ & 0.027453 & 0.026327 & 0.027295 & 0.027451 & 0.026327 & 0.027295 \\
\hline $\mathrm{CCl} 2=\mathrm{CHF}$ & $\mathrm{C}_{\mathrm{S}}$ & 0.025948 & 0.025066 & 0.025637 & 0.025952 & 0.025066 & 0.025637 \\
\hline $\mathrm{CFCl}=\mathrm{CH} 2$ & $\mathrm{C}_{\mathrm{S}}$ & 0.034078 & 0.032717 & 0.033167 & 0.034078 & 0.032717 & 0.033168 \\
\hline $\mathrm{Z}-\mathrm{CHCl}=\mathrm{CHF}$ & $\mathrm{C}_{\mathrm{S}}$ & 0.034759 & 0.033571 & 0.034381 & 0.034759 & 0.033571 & 0.034381 \\
\hline $\mathrm{E}-\mathrm{CHCl}=\mathrm{CHF}$ & $\mathrm{C}_{\mathrm{S}}$ & 0.034403 & 0.033593 & 0.034085 & 0.034403 & 0.033593 & 0.034090 \\
\hline
\end{tabular}

\footnotetext{
*geometry of neutral and the coupled nuclei estructures, the positive relaxed nuclei structure have $\mathrm{D}_{2}$ geometry
} 
Table S3. calculated Hartree Fock energies

\begin{tabular}{|c|c|c|c|c|c|c|}
\hline \multirow[b]{2}{*}{ substance } & \multicolumn{3}{|c|}{$6-311+G(3 d f, p)$} & \multicolumn{3}{|c|}{ GTLarge } \\
\hline & $\begin{array}{c}\mathrm{E}(\text { positive } \\
\text { coupled nuclei }) / \\
\text { Hartree }\end{array}$ & $\begin{array}{l}\mathrm{E} \text { (positive relaxed } \\
\text { nuclei) / Hartree }\end{array}$ & $\begin{array}{c}\mathrm{E}(\text { neutral)/ } \\
\text { Hartree }\end{array}$ & $\begin{array}{c}\mathrm{E}(\text { positive } \\
\text { coupled nuclei }) / \\
\text { Hartree }\end{array}$ & $\begin{array}{l}\mathrm{E} \text { (positive relaxe } \\
\text { nuclei) / Hartree }\end{array}$ & $\begin{array}{c}\text { E(neutral)/ } \\
\text { Hartree }\end{array}$ \\
\hline $\mathrm{CH} 2=\mathrm{CH} 2$ & -77.7345328800 & -77.73613374 & -78.0619988838 & -77.7345363912 & -77.73605307 & -78.0620576666 \\
\hline $\mathrm{CH} 2=\mathrm{CHCl}$ & -536.665443418 & -536.67464786 & -536.992991789 & -536.666214075 & -536.67530620 & -536.993590604 \\
\hline $\mathrm{E}-\mathrm{CHCl}=\mathrm{CHCl}$ & -995.594541776 & -995.60514101 & -995.919329828 & -995.596028735 & -995.60649905 & -995.920491266 \\
\hline $\mathrm{Z}-\mathrm{CHCl}=\mathrm{CHCl}$ & -995.593984993 & -995.60421680 & -995.919228233 & -995.595343264 & -995.60545437 & -995.920245420 \\
\hline $\mathrm{E}-\mathrm{CHCl}=\mathrm{CFCl}$ & -1094.46855934 & -1094.48396505 & -1094.79910760 & -1094.46838976 & -1094.48359679 & -1094.79882879 \\
\hline $\mathrm{Z}-\mathrm{CHCl}=\mathrm{CFCl}$ & -1094.46747933 & -1094.48324616 & -1094.79781748 & -1094.46721356 & -1094.48281590 & -1094.79752101 \\
\hline $\mathrm{CH} 2=\mathrm{CHF}$ & -176.611801445 & -176.62663201 & -176.949760302 & -176.610171633 & -176.62473256 & -176.948471905 \\
\hline $\mathrm{Z}-\mathrm{CHF}=\mathrm{CHF}$ & -275.480295817 & -275.50354685 & -275.827468011 & -275.477005972 & -275.49988650 & -275.824715041 \\
\hline $\mathrm{E}-\mathrm{CHF}=\mathrm{CHF}$ & -275.480071972 & -275.50366418 & -275.825850761 & -275.476694993 & -275.49992598 & -275.823191524 \\
\hline $\mathrm{E}-\mathrm{CFCl}=\mathrm{CHF}$ & -734.409485640 & -734.43180216 & -734.750622910 & -734.406908685 & -734.42896127 & -734.748433749 \\
\hline $\mathrm{Z}-\mathrm{CFCl}=\mathrm{CHF}$ & -734.409970403 & -734.43227063 & -734.750438726 & -734.407389303 & -734.42941187 & -734.748266157 \\
\hline $\mathrm{E}-\mathrm{CFCl}=\mathrm{CFCl}$ & -1193.33695353 & -1193.35725122 & -1193.67231693 & -1193.33507304 & -1193.35515673 & -1193.67061146 \\
\hline $\mathrm{Z}-\mathrm{CFCl}=\mathrm{CFCl}$ & -1193.33518142 & -1193.35578362 & -1193.67087158 & -1193.33328833 & -1193.35369448 & -1193.66918550 \\
\hline $\mathrm{CF} 2=\mathrm{CFCl}$ & -833.285002641 & -833.31262652 & -833.632775615 & -833.280417963 & -833.30771528 & -833.628846057 \\
\hline $\mathrm{CF} 2=\mathrm{CF} 2$ & -473.228045071 & -473.26520489 & -473.591332165 & -473.220591124 & -473.25726987 & -473.585152603 \\
\hline $\mathrm{CCl} 2=\mathrm{CCl} 2$ & -1913.43552600 & -1913.44587226 & -1913.75560034 & -1913.43738085 & -1913.44763532 & -1913.75714011 \\
\hline $\mathrm{CCl} 2=\mathrm{CF} 2$ & -1193.34232170 & -1193.36258797 & -1193.67921345 & -1193.33979907 & -1193.35978304 & -1193.67695361 \\
\hline $\mathrm{CCl} 2=\mathrm{CHCl}$ & -1454.51684121 & -1454.52756974 & -1454.83943745 & -1454.51850169 & -1454.52910585 & -1454.84077107 \\
\hline $\mathrm{CF} 2=\mathrm{CHF}$ & -374.357497013 & -374.38848194 & -374.712250534 & -374.352130807 & -374.38264040 & -374.707830282 \\
\hline $\mathrm{CCl} 2=\mathrm{CFCl}$ & -1553.38833198 & -1553.40307886 & -1553.71527961 & -1553.38835731 & -1553.40293590 & -1553.71520687 \\
\hline $\mathrm{CCl} 2=\mathrm{CH} 2$ & -995.589637654 & -995.60068649 & -995.916108211 & -995.590749554 & -995.60165009 & -995.917033750 \\
\hline $\mathrm{CF} 2=\mathrm{CH} 2$ & -275.495437817 & -275.51892538 & -275.842542724 & -275.491840947 & -275.51487593 & -275.839588897 \\
\hline $\mathrm{CHCl}=\mathrm{CF} 2$ & -734.421646878 & -734.44334349 & -734.762715606 & -734.418824705 & -734.44018773 & -734.760222851 \\
\hline $\mathrm{CCl} 2=\mathrm{CHF}$ & -1094.46367051 & -1094.47934617 & -1094.79432892 & -1094.46309908 & -1094.47856831 & -1094.79380915 \\
\hline $\mathrm{CFCl}=\mathrm{CH} 2$ & -635.541296295 & -635.55773256 & -635.876670245 & -635.540456027 & -635.55665796 & -635.875958952 \\
\hline $\mathrm{Z}-\mathrm{CHCl}=\mathrm{CHF}$ & -635.540671255 & -635.55607983 & -635.875014327 & -635.539793736 & -635.55497862 & -635.874165835 \\
\hline $\mathrm{E}-\mathrm{CHCl}=\mathrm{CHF}$ & -635.540052642 & -635.55605189 & -635.873800018 & -635.539164716 & -635.55495469 & -635.873050704 \\
\hline
\end{tabular}


Table S4: experimental AIP and VIP from literature, values in $\mathrm{eV}$.

\begin{tabular}{|c|c|c|}
\hline molecule & AIP experimental & VIP experimental \\
\hline \multirow[t]{22}{*}{$\mathrm{CH}_{2}=\mathrm{CH}_{2}$} & $\sim 10.5^{a}$ & $10.68^{a}$ \\
\hline & $10.5138 \pm 0.0006^{b, c, d}$ & $10.50 \pm 0.01^{a}$ \\
\hline & $10.51 \pm 0.015^{e}$ & $10.5^{a}$ \\
\hline & $10.509 \pm 0.005^{e}$ & \\
\hline & $10.51^{a, f, g, c}$ & \\
\hline & $10.50 \pm 0.02^{a, f, g}$ & \\
\hline & $10.514 \pm 0.007^{a}$ & \\
\hline & $10.51 \pm 0.02^{a, f, g}$ & \\
\hline & $10.517 \pm 0.002^{a}$ & \\
\hline & $10.517 \pm 0.003^{a}$ & \\
\hline & $10.5^{a}$ & \\
\hline & $10.507 \pm 0.004^{a, g . f}$ & \\
\hline & $10.51 \pm 0.01^{a}$ & \\
\hline & $10.515 \pm 0.003^{a}$ & \\
\hline & $10.56^{a}$ & \\
\hline & $10.51 \pm 0.05^{f, g}$ & \\
\hline & $10.50 \pm 0.05^{f, g}$ & \\
\hline & $10.511 \pm 0.005^{f, g}$ & \\
\hline & $10.50 \pm 0.01^{f, g}$ & \\
\hline & $10.52 \pm 0.01^{f, g}$ & \\
\hline & $10.51 \pm 0.03^{f, g}$ & \\
\hline & $10.80 \pm 0.05^{f, g}$ & \\
\hline \multirow[t]{7}{*}{$\mathrm{CHCl}=\mathrm{CH}_{2}$} & $9.98 \pm 0.02^{c}$ & $10.2^{\mathrm{e}}$ \\
\hline & $9.99 \pm 0.02^{a . b, d}$ & $10.15^{a}$ \\
\hline & $10.01^{a}$ & \\
\hline & $10.00^{f g}$ & \\
\hline & $10.00 \pm 0.01^{f, g}$ & \\
\hline & $10.00 \pm 0.02^{f, g}$ & \\
\hline & $9.995^{f, g}$ & \\
\hline
\end{tabular}


Table S4: Continuation

\begin{tabular}{|c|c|c|}
\hline molecule & AIP experimental & VIP experimental \\
\hline \multirow[t]{8}{*}{ (E) $-\mathrm{CHCl}=\mathrm{CHCl}$} & $9.64^{f, g}$ & $9.8^{e}$ \\
\hline & $9.69^{f, g}$ & $9.80^{a}$ \\
\hline & $\sim 9.6^{f, g}$ & $11.92^{a}$ \\
\hline & $9.66 \pm 0.03^{f, g}$ & $9.72^{a}$ \\
\hline & $9.64 \pm 0.02^{b, f, d, g}$ & \\
\hline & $9.63^{f, g}$ & \\
\hline & $9.6306 \pm 0.0006^{h}$ & \\
\hline & $9.63097 \pm 0.00025^{i}$ & \\
\hline \multirow[t]{8}{*}{ (Z)- $\mathrm{CHCl}=\mathrm{CHCl}$} & $9.65^{f, g}$ & $9.8^{e}$ \\
\hline & $9.68^{f, g}$ & $9.80^{a}$ \\
\hline & $9.66^{f, g}$ & \\
\hline & $9.65 \pm 0.01^{f, g}$ & \\
\hline & $9.66 \pm 0.02^{f, g}$ & \\
\hline & $9.65^{f, g}$ & \\
\hline & $9.66 \pm 0.01^{b, d}$ & \\
\hline & $9.65839 \pm 0.00025^{j}$ & \\
\hline \multirow[t]{5}{*}{$\mathrm{CCl}_{2}=\mathrm{CH}_{2}$} & $9.83^{f, g}$ & $10.0^{e}$ \\
\hline & $9.74^{f, g}$ & $9.99 \pm 0.02^{a}$ \\
\hline & $9.86^{f, g}$ & $10.00^{a}$ \\
\hline & $9.79^{f, g}$ & \\
\hline & $9.81 \pm 0.04^{b, d, f}$ & \\
\hline \multirow[t]{6}{*}{$\mathrm{CCl}_{2}=\mathrm{CHCl}$} & $9.68^{a}$ & $9.6^{e}$ \\
\hline & $9.48^{f, g}$ & \\
\hline & $9.45 \pm 0.01^{f, g}$ & \\
\hline & $9.45^{f, g}$ & \\
\hline & $9.47 \pm 0.01^{f, g}$ & \\
\hline & $9.46 \pm 0.02^{d}$ & \\
\hline
\end{tabular}


Table S4: Continuation

\begin{tabular}{|c|c|c|}
\hline molecule & AIP experimental & VIP experimental \\
\hline \multirow[t]{6}{*}{$\mathrm{CCl}_{2}=\mathrm{CCl}_{2}$} & $9.3256 \pm 0.0006^{c}$ & $9.5^{e}$ \\
\hline & $9.51^{a}$ & \\
\hline & $9.34^{f, g}$ & \\
\hline & $9.32 \pm 0.01^{f, g}$ & \\
\hline & $9.32^{f, g}$ & \\
\hline & $9.326 \pm 0.001^{b, d}$ & \\
\hline \multirow[t]{9}{*}{$\mathrm{CH}_{2}=\mathrm{CHF}$} & $10.36 \pm 0.02^{a}$ & $10.63 \pm 0.02^{a}$ \\
\hline & $10.3^{a}$ & $10.56 \pm 0.02^{a}$ \\
\hline & $10.36^{a}$ & \\
\hline & $10.363 \pm 0.015^{a}$ & \\
\hline & $10.37^{a}$ & \\
\hline & $10.35 \pm 0.01^{f a}$ & \\
\hline & $10.37^{f, g}$ & \\
\hline & $10.37 \pm 0.02^{f, g}$ & \\
\hline & $10.36 \pm 0.01^{b, d}$ & \\
\hline \multirow[t]{4}{*}{ (E) $-\mathrm{CHF}=\mathrm{CHF}$} & $10.15^{e}$ & $10.63 \pm 0.02^{a}$ \\
\hline & $10.21 \pm 0.02^{a}$ & $10.38 \pm 0.02^{a}$ \\
\hline & $10.15 \pm 0.02^{a}$ & $10.39 \pm 0.02^{a}$ \\
\hline & $10.21^{a . g}$ & $10.38^{a}$ \\
\hline \multirow[t]{6}{*}{ (Z) $-\mathrm{CHF}=\mathrm{CHF}$} & $10.20^{e}$ & $10.62 \pm 0.02^{\mathrm{a}}$ \\
\hline & $10.1^{a}$ & $10.44 \pm 0.02^{\mathrm{a}}$ \\
\hline & $10.23 \pm 0.02^{a . b}$ & $10.41 \pm 0.02^{\mathrm{a}}$ \\
\hline & $10.20 \pm 0.02^{a}$ & $10.43^{\mathrm{a}}$ \\
\hline & $10.23^{a}$ & \\
\hline & $10.2^{d}$ & \\
\hline
\end{tabular}


Table S4: Continuation

\begin{tabular}{|c|c|c|}
\hline molecule & AIP experimental & VIP experimental \\
\hline \multirow[t]{7}{*}{$\mathrm{CF}_{2}=\mathrm{CH}_{2}$} & $10.29^{e}$ & $10.70 \pm 0.02^{a}$ \\
\hline & $10.29 \pm 0.02^{a}$ & $10.69 \pm 0.02^{a}$ \\
\hline & $10.29^{a}$ & \\
\hline & $10.29 \pm 0.01^{a . b, d}$ & \\
\hline & $10.31^{f, g}$ & \\
\hline & $10.31 \pm 0.02^{f, g}$ & \\
\hline & $10.30^{f, g}$ & \\
\hline \multirow[t]{3}{*}{$\mathrm{CHF}=\mathrm{CF}_{2}$} & $10.14 \pm 0.02^{a}$ & $10.62 \pm 0.02^{a}$ \\
\hline & $10.14^{a, f, g, d}$ & $10.54 \pm 0.02^{a}$ \\
\hline & & $10.53^{a}$ \\
\hline \multirow[t]{10}{*}{$\mathrm{CF}_{2}=\mathrm{CF}_{2}$} & $10.114 \pm 0.010^{c}$ & $10.69 \pm 0.02^{a}$ \\
\hline & $10.14 \pm 0.02^{a}$ & $10.56 \pm 0.02^{a}$ \\
\hline & $10.14^{a}$ & $10.52^{a}$ \\
\hline & $10.32^{a}$ & \\
\hline & $10.10^{a}$ & \\
\hline & $10.11^{f, g}$ & \\
\hline & $10.12 \pm 0.01^{f, g}$ & \\
\hline & $10.12^{f, g}$ & \\
\hline & $10.12 \pm 0.02^{b}$ & \\
\hline & $10.14 \pm 0.07^{d}$ & \\
\hline \multirow[t]{2}{*}{ (E) $-\mathrm{CHCl}=\mathrm{CHF}$} & $9.87 \pm 0.02^{f, k, g}$ & \\
\hline & $9.87 \pm 0.01^{f, k, g}$ & \\
\hline \multirow[t]{2}{*}{ (Z) $-\mathrm{CHCl}=\mathrm{CHF}$} & $9.86 \pm 0.02^{f, k, g}$ & \\
\hline & $9.87 \pm 0.01^{f, k, g}$ & \\
\hline \multirow[t]{2}{*}{$\mathrm{CH}_{2}=\mathrm{CFCl}$} & $10.7 \pm 0.2^{a}$ & \\
\hline & $9.97^{a}$ & \\
\hline (E) $-\mathrm{CFCl}=\mathrm{CHF}$ & $9.83 \pm 0.02^{f, g}$ & \\
\hline
\end{tabular}


Table S4: Continuation

\begin{tabular}{lll}
\hline molecule & AIP experimental & VIP experimental \\
\hline (Z)-CFCl=CHF & $9.86 \pm 0.02^{f, g}$ & \\
$\mathrm{CF}_{2}=\mathrm{CHCl}$ & $9.76^{a}$ \\
& $9.84^{f, g}$ \\
& $9.80 \pm 0.04^{d, b}$ & \\
& $10.6 \pm 0.1^{a}$ \\
$\mathrm{CF}_{2}=\mathrm{CFCl}$ & $9.76^{a}$ \\
& $9.82^{a}$ \\
& $9.84^{f, g}$ \\
& $9.81 \pm 0.26^{a}$ \\
& $10.2 \pm 0.3^{a, f}$ \\
$\mathrm{CFCl}^{\mathrm{d}}=\mathrm{CFCl}$ & $9.62^{a}$ \\
& $9.69 \pm 0.01^{a}$ \\
$\mathrm{CF}_{2}=\mathrm{CCl}{ }_{2}$ & $9.65^{f, g}$ \\
& $9.65 \pm 0.03^{d}$
\end{tabular}

a: Lias. S. G.; Levin. R. D.; Kafafi. S. A. In "Ion Energetics Data" in NIST Chemistry WebBook. NIST Standard Reference Database Number 69; Linstrom. P. J.. Mallard. W. G.. Eds.; National Institute of Standards and Technology: Gaithersburg MD. June 2005 (http://webbook.nist.gov).

b: Lide CRC Handbook of Chemistry and Physics. 79 ed; CRC Press: Boca Raton. 1998-1999.

c: Lias. S. G.; Liebman. J. F. In "Ion Energetics Data" in NIST Chemistry WebBook. NIST Standard Reference Database Number 69. Linstrom. P. J.. Mallard. W. G.. Eds.; National Institute of Standards and Technology: Gaithersburg MD. June 2005 (http://webbook.nist.gov).

d: Lias. S. G. In "Ion Energetics Data" in NIST Chemistry WebBook. NIST Standard Reference Database Number 69. Linstrom. P. J.. Mallard. W. G.. Eds.; National Institute of Standards and Technology: Gaithersburg MD. June 2005 (http://webbook.nist.gov).

e: Lias. S. G.; Bartmess. J. E.; Liebman. J. F.; Holmes. J. L.; Levin. R. D.; Mallard. W. G. In "Ion Energetics Data" in NIST Chemistry WebBook. NIST Standard Reference Database Number 69. Linstrom. P. J.. Mallard. W. G.. Eds.; National Institute of Standards and Technology: Gaithersburg MD. June 2005 (http://webbook.nist.gov).

f: Lias. S. G.; Bartmess. J. E.; Liebman. J. F.; Holmes. J. L.; Levin. R. D.; Mallard. W. G. Gas-Phase Ion and Neutral Thermochemistry. J. Phys. Chem. Ref. Data. 1988. 17 (supl. 1).

g: Rosenstock. H.M.; Draxl. K.; Steiner. B.W.; Herron. J.T. In "Ion Energetics Data" in NIST Chemistry WebBook. NIST Standard Reference Database Number 69; Linstrom. P. J.. Mallard. W. G.. Eds.; National Institute of Standards and Technology: Gaithersburg MD. June 2005 (http://webbook.nist.gov)

h: Bae. Y. J; Lee. M.; Kim. M.S.J. Phys. Chem. A., 2006, 110, 8535.

i: Woo. H. K.; Wang. P.; Lau, K. C.; Xing. X.; Ng. C. Y. J. Phys. Chem. A., 2004, 108, 9637.

j: Lau. K. -C.; Woo. H. K.; Wang. P.; Xing. X.; Ng. C. Y. J. Chem. Phys., 2006, 124, 224311.

k: Rosenstock. H.M.; Draxl. K.; Steiner. B.W.; Herron. J.T. Energetics of Gaseous Ions. Journal of Physical and Chemical Reference Data. 1977. 6 (supl. 1) 


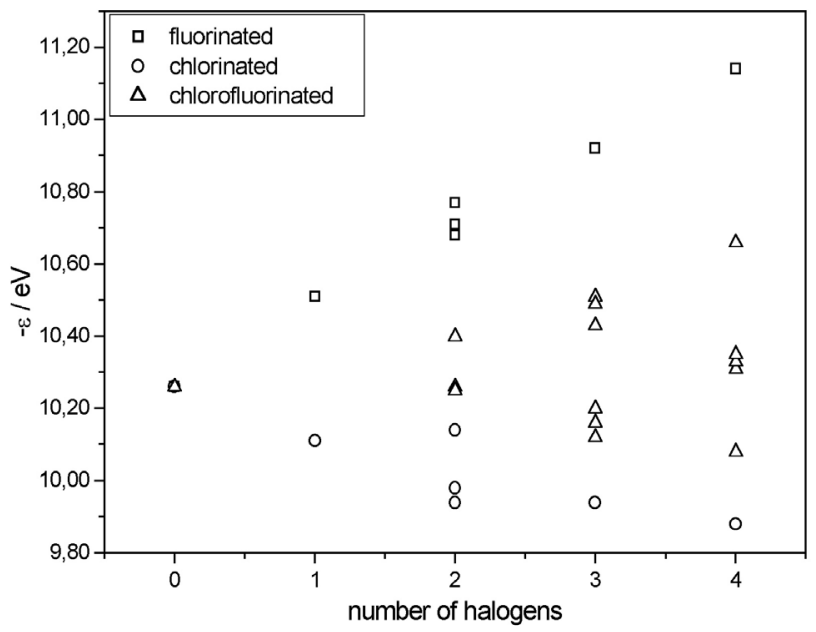

Figure S1: HOMO energy as a function of the number of halogens (G2 theory results)

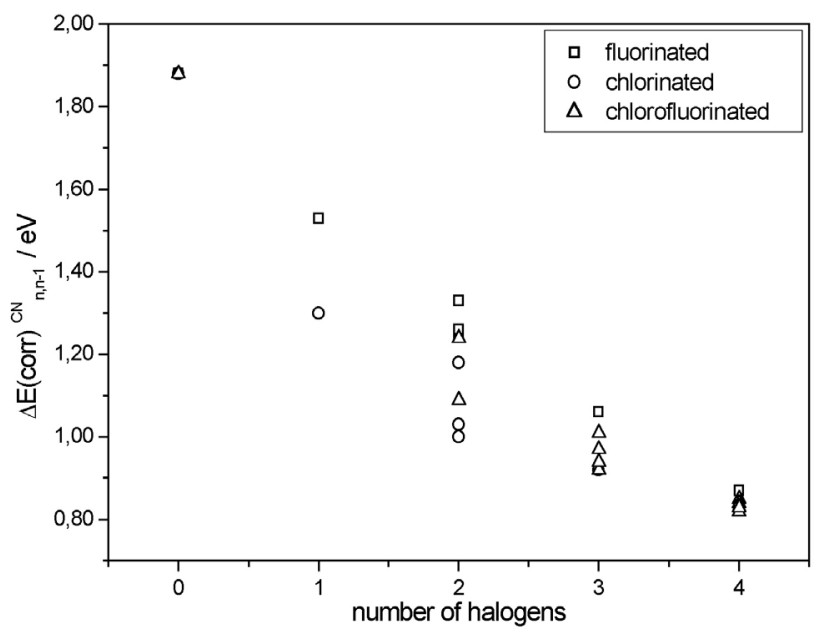

Figure S3: $\Delta \mathrm{E}(\operatorname{corr})_{n, \mathrm{n}-1}^{\mathrm{CN}}$ as a function of the number of halogens (G2 theory results)

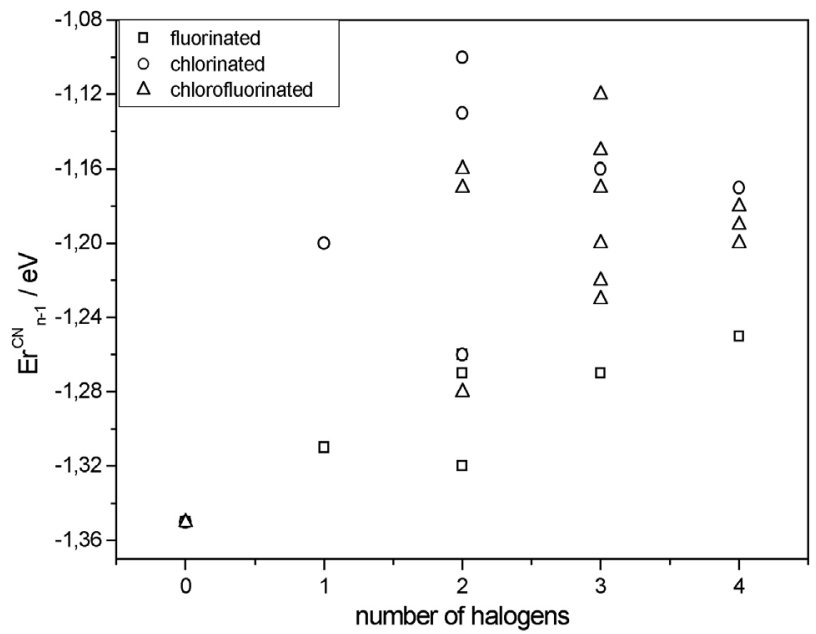

Figure $\mathrm{S} 2: \mathrm{Er}_{\mathrm{n}-1}^{\mathrm{CN}}$ as a function of the number of halogens (G2 theory results)

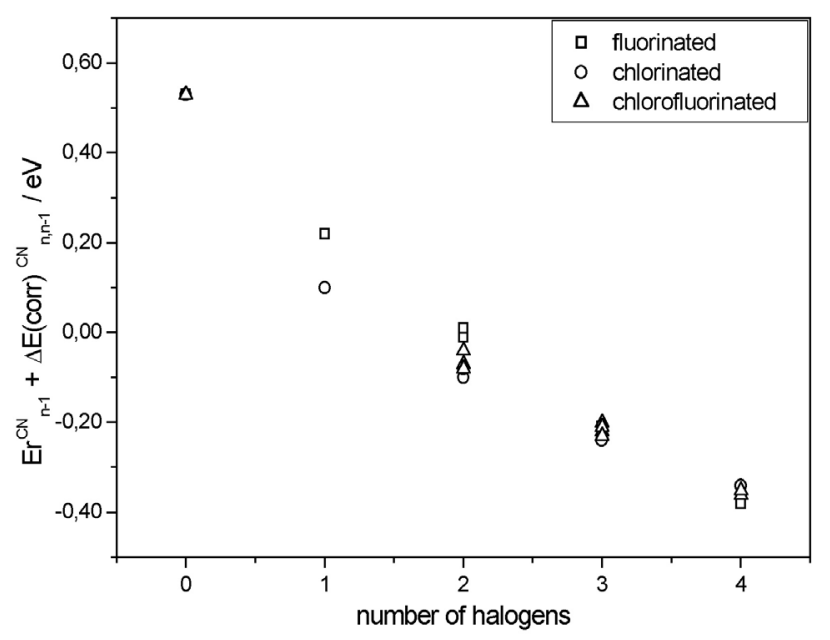

Figure $\quad \mathrm{S} 4: \quad \mathrm{Er}_{\mathrm{n}-1}^{\mathrm{CN}}+\Delta \mathrm{E}(\text { corr })_{\mathrm{n}, \mathrm{n}-1}^{\mathrm{CN}}$ as $\quad \mathrm{a}$ function of the number of halogens (G2 theory results) 


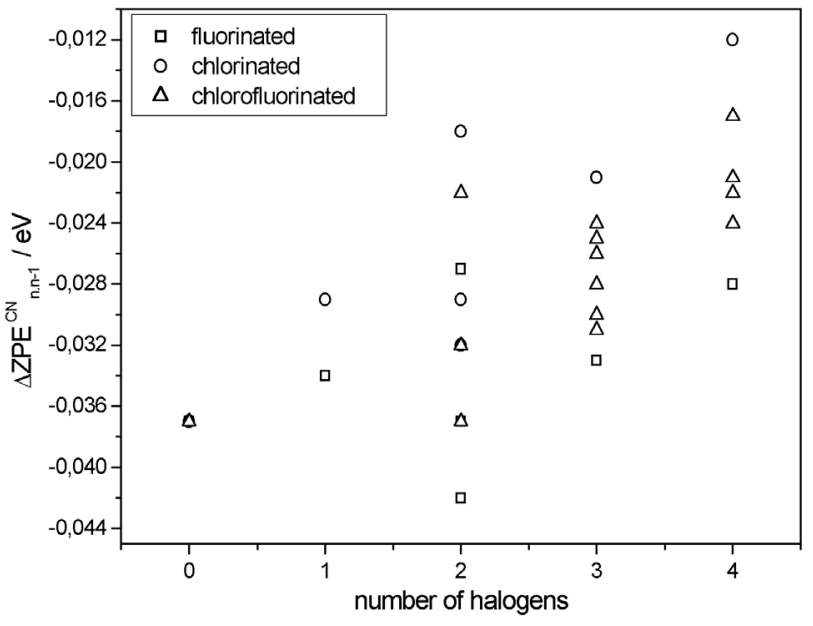

Figure S5: $\triangle \mathrm{ZPE}_{\mathrm{n}, \mathrm{n}-1}^{\mathrm{CN}}$ as a function of the number of halogens ( $\mathrm{G} 2$ theory results)

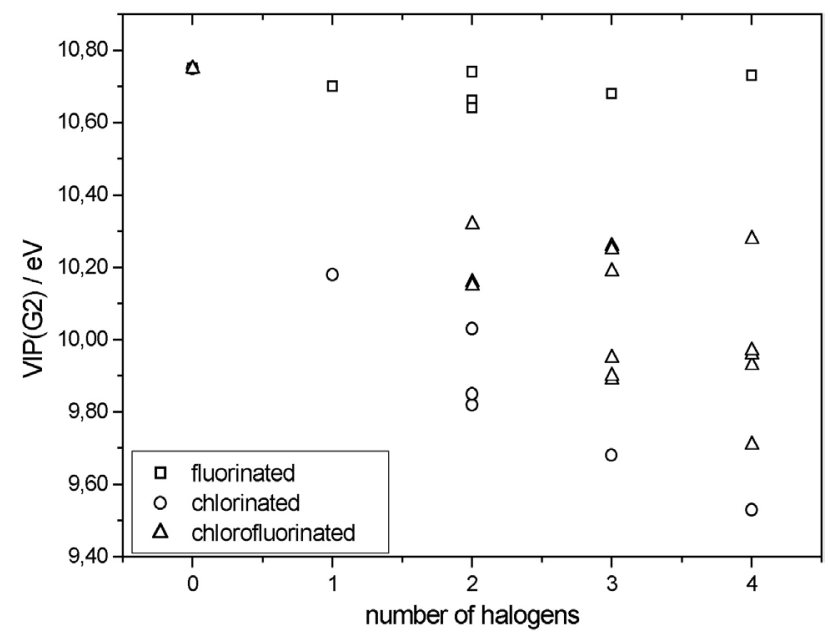

Figure S7: $\operatorname{VIP}(\mathrm{G} 2)$ as a function of the number of halogens (G2 theory results)

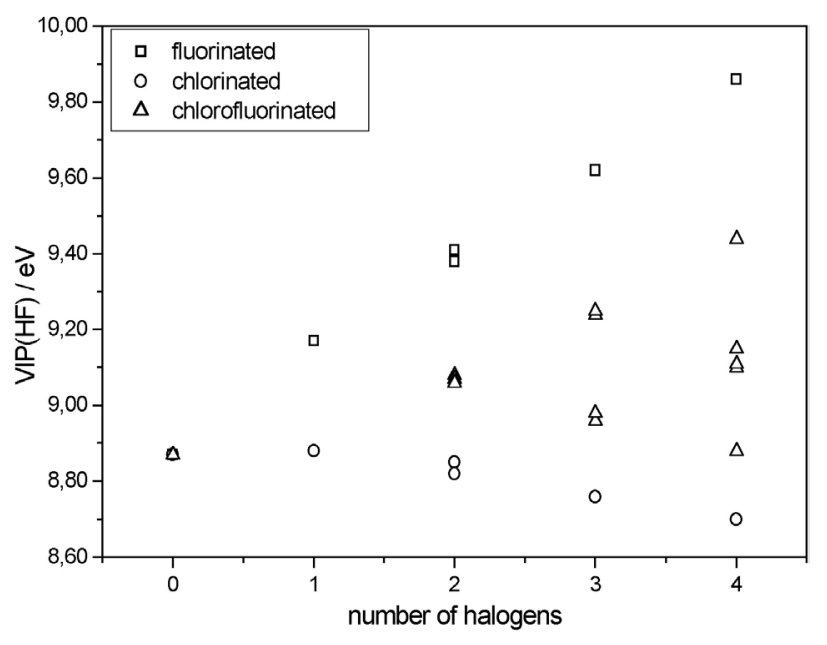

Figure S6: VIP(HF) as a function of the number of halogens (G2 theory results)

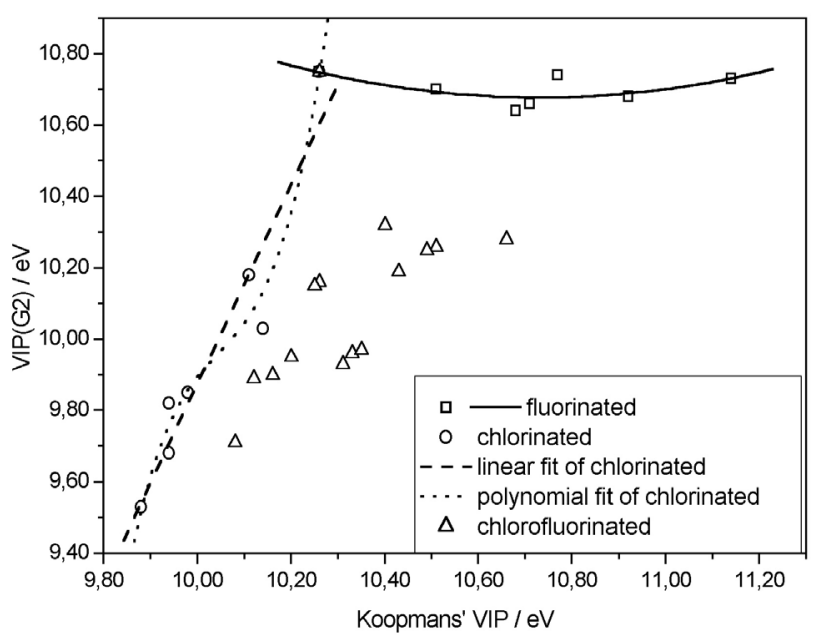

Figure S8: $\operatorname{VIP}(\mathrm{G} 2)$ as a function of the HOMO energy (G2 theory results). Polynomial fit to fluorethenes is $2^{\text {nd }}$ order. polynomial fit to chloroethenes is $3^{\text {rd }}$ order. 


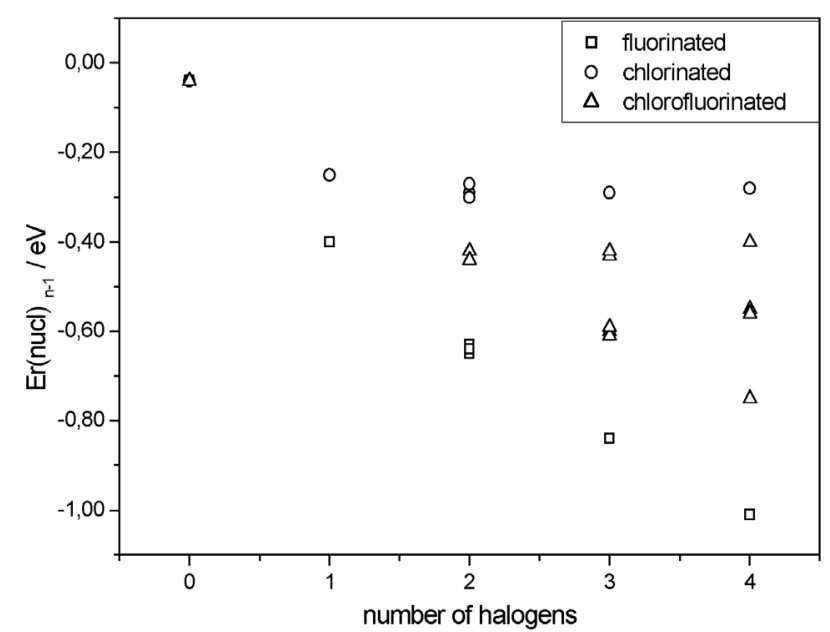

Figure S9: $\operatorname{Er}(\text { nucl })_{n-1}$ as a function of the number of halogens ( $\mathrm{G} 2$ theory results)

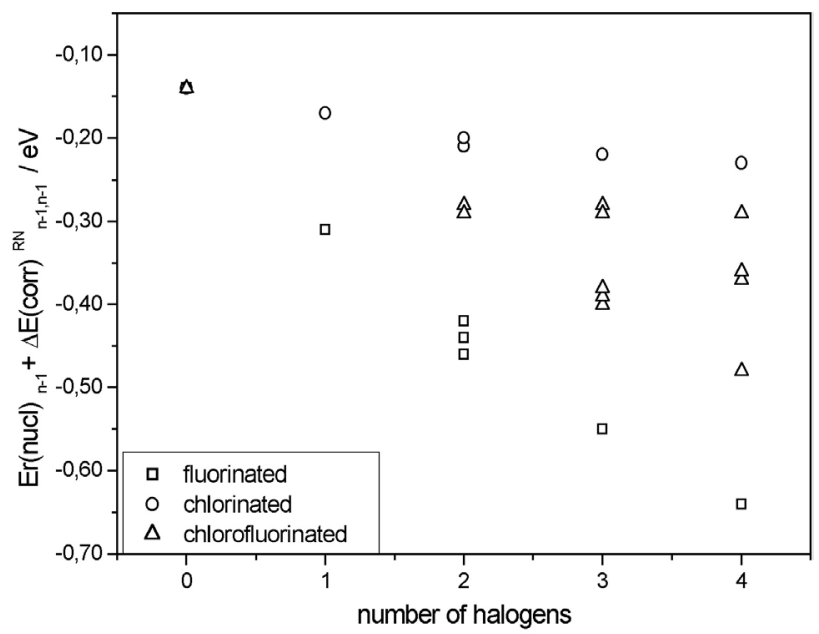

Figure S11: $E_{r}(\text { nucl })_{n-1}+\Delta E(\text { corr })_{n-1, n-1}^{R N}$ as a function of the number of halogens (G2 theory results)

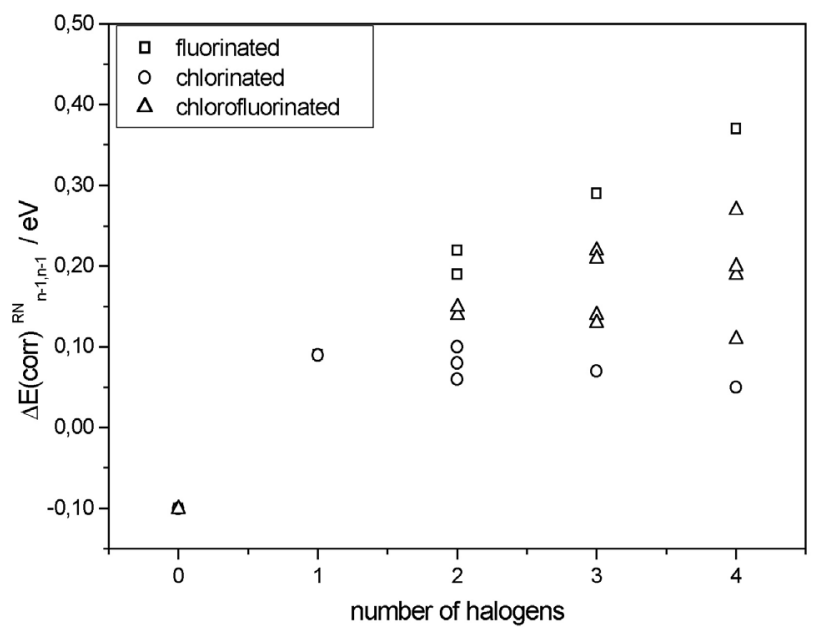

Figure S10: $\Delta \mathrm{E}(\operatorname{corr})_{\mathrm{n}-1, \mathrm{n}-1}^{\mathrm{RN}}$ as a function of the number of halogens ( $\mathrm{G} 2$ theory results)

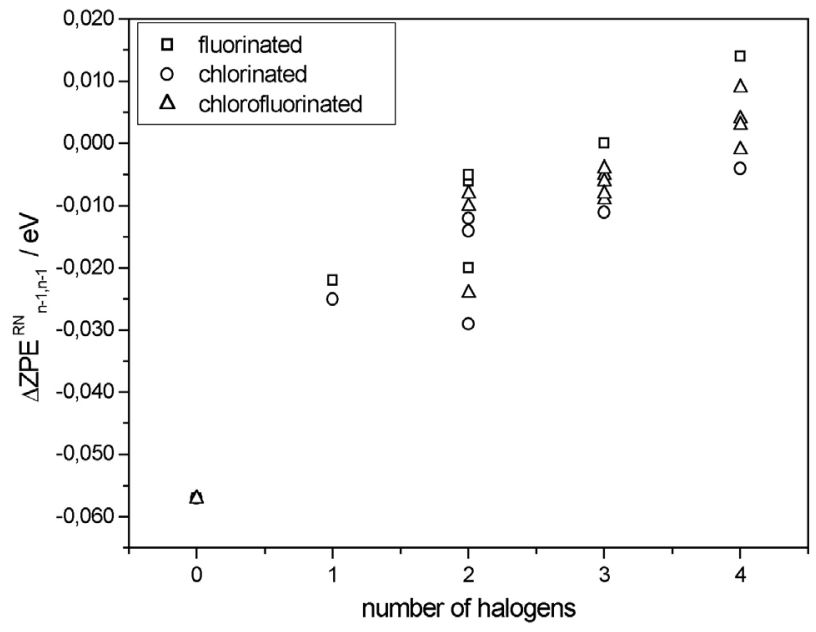

Figure S12: $\triangle Z P E_{n-1, n-1}^{R N}$ as a function of the number of halogens (G2 theory results) 


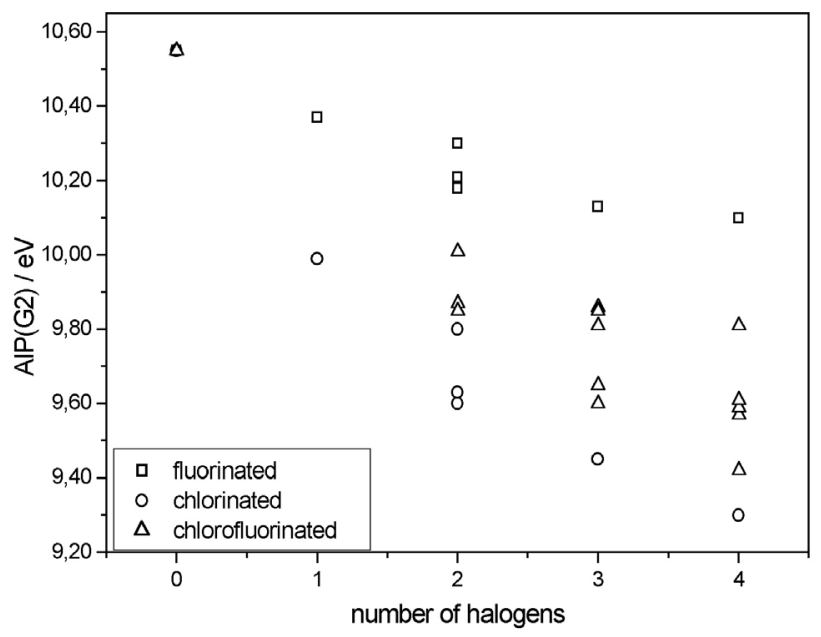

Figure S13: $\mathrm{AIP}(\mathrm{G} 2)$ as a function of the number of halogens (G2 theory results)

\section{Complete citation for reference 13:}

Frisch. M. J.; Trucks. G. W.; Schlegel. H. B.; Scuseria. G. E.; Robb. M. A.; Cheeseman. J. R.; Zakrzewski. V. G.; Montgomery. J. A.. Jr.; Stratmann. R. E.; Burant. J. C.; Dapprich. S.; Millam. J. M.; Daniels. A. D.; Kudin. K. N.; Strain. M. C.; Farkas. O.; Tomasi. J.; Barone. V.; Cossi. M; Cammi. R.; Mennucci. B.; Pomelli. C.; Adamo. C.; Clifford. S.; Ochterski. J.; Petersson. G. A.; Ayala. P. Y.; Cui. Q.; Morokuma. K.; Malick. D. K.; Rabuck. A. D.; Raghavachari. K.; Foresman. J. B.; Cioslowski. J.; Ortiz. J. V.; Baboul. A. G.; Stefanov. B. B.; Liu. G.; Liashenko. A.; Piskorz. P.; Komaromi. I.; Gomperts. R.; Martin. R. L.; Fox. D. J.; Keith. T.; Al-Laham. M. A.; Peng. C. Y.; Nanayakkara. A.; Challacombe. M.; Gill. P. M. W.; Johnson. B.; Chen. W.; Wong. M. W.; Andres. J. L.; Gonzalez. C.; Head-Gordon. M.; Replogle. E. S.; Pople. J. A. Gaussian 98. Revision A.9; Gaussian. Inc.: Pittsburgh. PA. 1998. 


\section{Complete citation for reference 14:}

Frisch. M. J.; Trucks. G. W.; Schlegel. H. B.; Scuseria. G. E.; Robb. M. A.; Cheeseman. J. R.; Montgomery. J. A.. Jr.; Vreven. T.; Kudin. K. N.; Burant. J. C.; Millam. J. M.; Iyengar. S. S.; Tomasi. J.; Barone. V.; Mennucci. B.; Cossi. M.; Scalmani. G.; Rega. N.; Petersson. G. A.; Nakatsuji. H.; Hada. M.; Ehara. M.; Toyota. K.; Fukuda. R.; Hasegawa. J.; Ishida. M.; Nakajima. T.; Honda. Y.; Kitao. O.; Nakai. H.; Klene. M.; Li. X.; Knox. J. E.; Hratchian. H. P.; Cross. J. B.; Adamo. C.; Jaramillo. J.; Gomperts. R.; Stratmann. R. E.; Yazyev. O.; Austin. A. J.; Cammi. R.; Pomelli. C.; Ochterski. J. W.; Ayala. P. Y.; Morokuma. K.; Voth. G. A.; Salvador. P.; Dannenberg. J. J.; Zakrzewski. V. G.; Dapprich. S.; Daniels. A. D.; Strain. M. C.; Farkas. O.; Malick. D. K.; Rabuck. A. D.; Raghavachari. K.; Foresman. J. B.; Ortiz. J. V.; Cui. Q.; Baboul. A. G.; Clifford. S.; Cioslowski. J.; Stefanov. B. B.; Liu. G.; Liashenko. A.; Piskorz. P.; Komaromi. I.; Martin. R. L.; Fox. D. J.; Keith. T.; Al-Laham. M. A.; Peng. C. Y.; Nanayakkara. A.; Challacombe. M.; Gill. P. M. W.; Johnson. B.; Chen. W.; Wong. M. W.; Gonzalez. C.; Pople. J. A. Gaussian 03. Revision B.04; Gaussian. Inc.: Pittsburgh. PA. 2003. 